\title{
Intravoxel Incoherent Motion of Colon Cancer Liver Metastases for the Assessment of Response to Antiangiogenic Treatment: Results from a Pilot Study
}

\author{
Ayşegül Öz $z^{a}$ Sadık Server ${ }^{a}$ Bedriye Koyuncu Sökmen ${ }^{a}$ Esat Namal ${ }^{b}$ \\ Nagihan İnan ${ }^{a}$ Numan Cem Balcl ${ }^{c}$

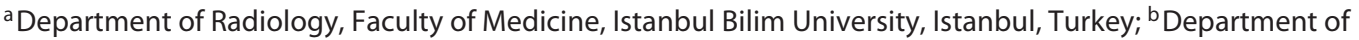 \\ Medical Oncology, Faculty of Medicine, Istanbul Bilim University, Istanbul, Turkey; ' Department of Radiology, \\ Cleveland Clinic, Lerner School of Medicine, Abu Dhabi, UAE
}

\section{Highlights of the Study}

- Intravoxel incoherent motion (IVIM) is a diffusion imaging method which is used clinically for the evaluation of tissue perfusion without the use of contrast media.

- The $\mathrm{f}$ (perfusion fraction) value of IVIM may quantitatively reflect the response of antiangiogenic therapy.

- IVIM could be added in routine protocols for magnetic resonance imaging to assess response of tumors to antiangiogenic agents.

\section{Keywords}

Colorectal carcinoma metastasis · Bevacizumab · Intravoxel incoherent motion · Diffusion-weighted MRI

\begin{abstract}
Objective: This study was aimed at evaluating the intravoxel incoherent motion (IVIM) parameter alterations of liver metastases of colorectal carcinoma (CRC) during antiangiogenic bevacizumab combination therapy. Methods: Twenty-five patients with CRC liver metastases treated with bevacizum$\mathrm{ab}$ in combination with FOLFOX-or-FOLFIRI protocols were enrolled in the study. MRI was performed using a 1.5-tesla scanner pre-treatment (PT) and at 3, 6, and 9 months of therapy. Routine abdominal MRI sequences and an IVIM-DWI
\end{abstract}

\begin{tabular}{ll}
\hline karger@karger.com & $\begin{array}{l}\text { C) } 2020 \text { The Author(s) } \\
\text { Published by S. Karger AG, Basel }\end{array}$ \\
www.karger.com/mpp & This is an Open Access article licensed under the Creative Commons \\
Karger & $\begin{array}{l}\text { Attribution-NonCommercial-4.0 International License (CC BY-NC) } \\
\text { (http://www.karger.com/Services/OpenAccessLicense), applicable to } \\
\text { the online version of the article only. Usage and distribution for com- } \\
\text { mercial purposes requires written permission. }\end{array}$
\end{tabular}

(diffusion-weighted imaging) sequence were obtained. The IVIM-DWI sequence was executed with 16 b-values varying from 0 to $1,400 \mathrm{~s} / \mathrm{mm}^{2}$. The mean values of apparent diffusion coefficient $(A D C)$, true diffusion (D), pseudodiffusion $\left(D^{*}\right)$, and perfusion fraction $(f)$ of each metastasis were obtained for all b-values, and the time-related changes were recorded to analyze the chronologic responses to antiangiogenic therapy. The RECIST 1.1 criteria were used for the evaluation of treatment response. Results: The diameters of the metastases diminished significantly at 9 months when compared with PT $(p=0.03)$. The D $(p=0.10)$ and ADC $(p=0.21)$ values of the metastases increased at 9 months of therapy. D* was the highest at 3 months $(p=0.24)$; it decreased at 6 ( $p=0.97)$ and 9 months $(p=0.87)$ of therapy. The f value had peaked at 3 months $(p=0.51)$ and started to decrease there- 
after. At 6 months, f decreased to the lowest values $(p=0.12)$. Conclusion: IVIM parameters, particularly the perfusion fraction, may quantitatively reflect the response to antiangiogenic treatment. The antiangiogenic response manifests after 3 months of therapy before the RECIST-related response.

(c) 2020 The Author(s)

Published by S. Karger AG, Basel

\section{Introduction}

Colorectal carcinoma (CRC) is the second most common cause of cancer death, and its most frequent distant metastatic target organ is the liver. The treatment of metastatic CRC (mCRC) has improved in the last 20 years. Thirion et al. [1] have reported that the addition of leucovorin to 5 -fluorouracil increased overall survival to 12 months in mCRC patients. This protocol and its combinations have become the cornerstones for development of new protocols for such patients [1]. Oxaliplatin and irinotecan combinations, known as FOLFOX and FOLFIRI protocols, improved the overall survival to 16-18 months. Moreover, targeted antiangiogenic agents have been added to these basic cornerstone protocols, increasing the survival by up to 24-30 months [2]. Bevacizumab, widely used for mCRC treatment, is a targeted antiangiogenic agent; it is an antibody to vascular endothelial growth factor. Its multiple mechanisms of action result in the inhibition of new vessel growth [3].

The response of mCRC patients to treatment can be evaluated by patient's physical examination, carcinoembryonic antigen levels if initially elevated, and various imaging techniques. Noninvasive imaging techniques play an important role in evaluating tumor responses during antiangiogenic therapies [4].

As antiangiogenic therapy mainly acts on the tumor vasculature, change in tumor size requires a long time. Therefore, biomarkers other than size are needed to assess the early response to therapy. The response to antiangiogenic therapy is assessed with the use of contrastenhanced imaging techniques using both CT and MRI [5]. However, contrast-induced nephropathy and nephrogenic systemic fibrosis are major risk factors in patients with diminished renal function. Cancer patients are particularly at risk of contrast-induced renal impairment [6].

Diffusion-weighted magnetic resonance imaging (DWI) has become a preferred diagnostic tool for the evaluation of liver lesions and parenchymal changes [7]. DWI reflects the water proton mobility in a voxel and provides information about cell microstructure, microcirculation, cell membrane integrity, and cell viability $[3,7]$. DWI measures the random motion of water molecules in the tumor. When the motion of water molecules is restricted due to high cellularity in the environment, their diffusion is also restricted. After the treatment, the cellularity of the tumor decreases, and in some cases necrosis occurs; these changes bring about an increase in the free motion of water molecules and an increase in their diffusion. DWI and apparent diffusion coefficient (ADC) maps are also affected by tissue perfusion of the microvascular capillary [8]. The intravoxel incoherent motion (IVIM) theory of Le Bihan et al. [9] states that increasing the number of b-values leads to a multiexponential signal drop within each voxel and enables quantitative parameters that measure tissue microcapillary perfusion. This helps to differentiate between true molecular diffusion and microcapillary perfusion [9], and thus is helpful for the diagnosis and followup of the hypervascular lesions. As tumor perfusion changes after antiangiogenic treatments, low and high bvalues reflect the changes in microcapillary tissue perfusion and also diffusivity without the need of contrast agent use. The perfusion-sensitive parameters perfusion fraction $(f)$ and pseudodiffusion coefficient $\left(D^{*}\right)$, and the diffusion-sensitive parameter, true diffusion coefficient (D), can be measured and mapped separately with biexponential analysis [10]. The perfusion parameters are also obtained by signal intensity (SI) curve biexponential analysis with increasing $b$-values by the same technique [8].

Bevacizumab therapy is widely used for brain gliomas, kidney, lung, colon, rectum, cervix, ovary, and fallopian tube cancers. Recent studies also investigated the changes of IVIM parameters and tumor response in non-small cell lung cancer and gliomas $[11,12]$. The value of IVIM for the assessment of the effectiveness of the early treatment has been published in a few reports. In this pilot study, which, to the best of our knowledge is the first, we aimed to evaluate time-related tumor response to long-term antiangiogenic bevacizumab therapy combined with FOLFOX or FOLFIRI protocols in unresectable mCRC patients with liver metastases using IVIM-DWI parameters to also determine the optimal time interval between the onset of therapy and assessment of antiangiogenic effect with the use of IVIM parameters.

\section{Subjects and Methods}

This study was carried out between March 2016 and August 2018. The single-center, open-label, nonrandomized, prospective study protocol was approved by the Institutional Review Board, Clinical Research Ethics Committee for human investigations. The 

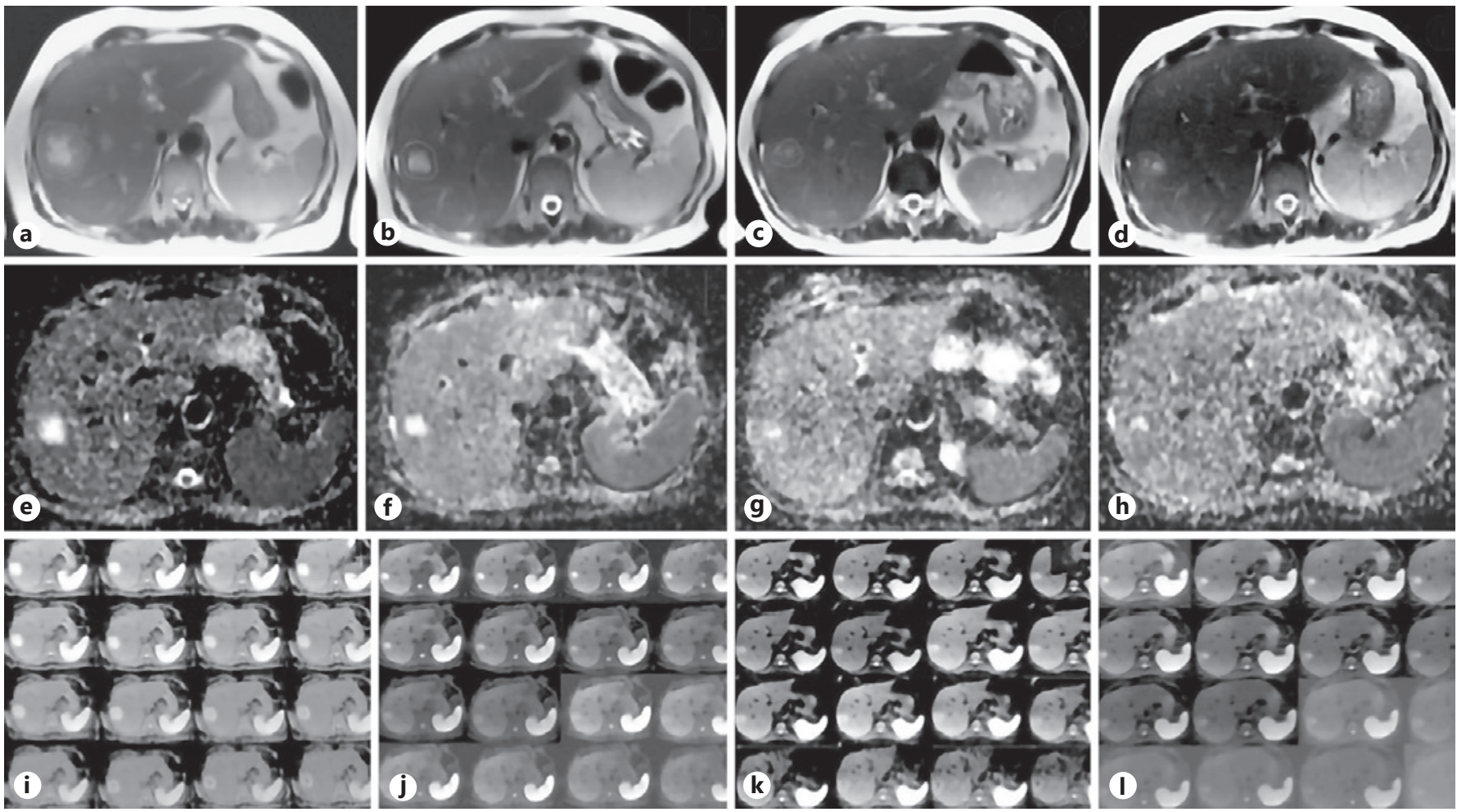

Fig. 1. Sixty-six-year-old female patient with stage IV metastatic colorectal carcinoma. a-d Liver metastases on T2-weighted axial images at PT and 3, 6, and 9 months of therapy, respectively. e-h ADC maps of the lesion. i-I IVIM images of the lesion with 16 different b-values (from 0 to $1,400 \mathrm{~s} / \mathrm{mm}^{2}$ ) at PT and 3, 6, and 9 months of therapy.

study was conducted according to the principles of the Declaration of Helsinki, and the International Conference on Harmonization - Good Clinical Practice. All participants provided written informed consent before they were included in the study.

\section{Patients}

Twelve patients diagnosed as having unresectable CRC with 25 liver metastases were enrolled in the study. Three patients (seven metastases) with poor diagnostic imaging quality due to motion artefacts were excluded from the study. One patient (two metastases) was lost to follow-up. Finally, 8 patients ( 3 females and 5 males) with a total of 16 metastases were included in this study. The mean age was 60 years, range $41-73$ years. All patients were treated for a total of 9 months using FOLFOX or FOLFIRI protocols combined with bevacizumab therapy.

\section{MR Imaging}

All patients underwent MRI before the treatment and then at 3,6 , and 9 months after the start of therapy. All MR images were obtained in a Siemens, Magnetom Symphony 1.5-tesla scanner (Erlangen, Germany) with a four-channel body coil. Routine precontrast upper abdomen MR images were acquired with the use of following imaging sequences: axial in- and out-of-phase (TR: 179, TE: 2.4-4.8, FA: 70) T1-weighted (T1W) turbo field echo and axial and coronal T2W single-shot turbo spin echo (TR: 1,350, TE:
92, TSE factor: 281, NEX: 1). Subsequently, respiratory-triggered IVIM and conventional DWI sequences were obtained with a single-shot echo-planar imaging sequence: IVIM with 16 different b-values of 0-100 (average 1), 200-300 (average 2), 400-800 (average 4), 900-1,000 (average 6), 1,100 (average 1), and 1,200-1,400 (average 6) $\mathrm{s} / \mathrm{mm}^{2}$. Three orthogonal diffusion-encoding directions were acquired.

T1W volumetric interpolated breath-hold examination (TR: 4.86, TE: 2.39, NEX: 1, FA: 10.0) sequences were obtained after administration of IV gadoterate meglumine (Gd-DOTA) $0.2 \mathrm{~mL} /$ kg (Dotarem; Guerbet LLC, NJ, USA). Post-contrast axial dynamic arterial, portal, venous, and delayed-phase images with fat suppression via the spectral adiabatic inversion recovery method were performed. The acquisition time for IVIM sequence was $10 \pm 3$ min, and 40-45 min for the total upper abdominal MRI. The images were acquired with a $5 \mathrm{~mm} / 1 \mathrm{~mm}$ slice thickness/intersection gap. Imaging matrix was $115 \times 192$. The field of view varied from 240 to $380 \mathrm{~mm}$ (Fig. 1).

\section{Image Analysis}

Two radiologists (A.O. and N.I. with, respectively, 12 and 16 years of experience in the evaluation of liver MRI) performed blinded assessment for the Response Evaluation Criteria in Solid Tumours (RECIST) analysis. After the longest diameters of the lesions were measured in the axial post-contrast images, evaluation 
Table 1. Quantitative analysis of IVIM parameters and comparison of pre-treatment values with values at 3, 6, and 9 months post-treatment in the 16 liver metastases

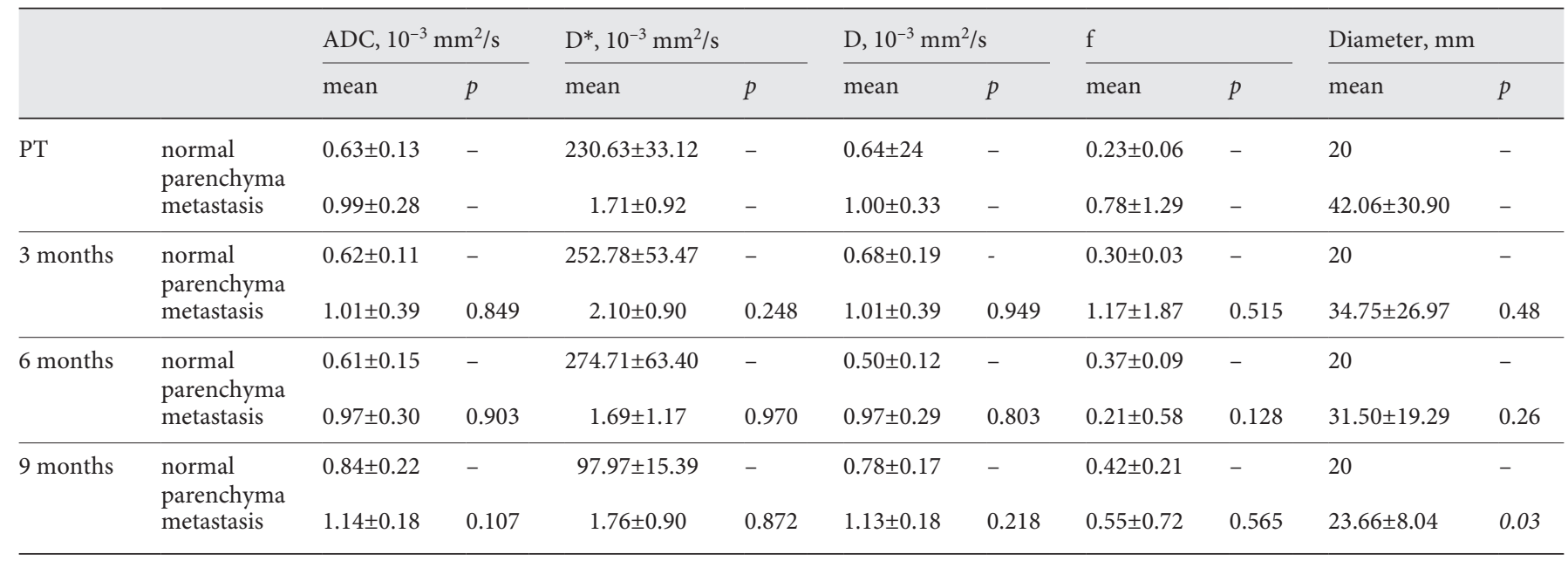

ADC, apparent diffusion coefficient; IVIM, intravoxel incoherent motion; $\mathrm{f}$, perfusion fraction ; $\mathrm{D}^{*}$, pseudodiffusion coefficient ; D, true diffusion coefficient; PT, pre-treatment.

was done according to the RECIST guidelines (version 1.1) [13]. Tumor response was classified as partial response (PR) when at least a $30 \%$ decrease in the sum of diameters of target lesions was detected. Tumor response was defined as progressive disease (PD) when at least a $20 \%$ increase in the smallest sum of diameters of target lesions with an absolute increase of at least $5 \mathrm{~mm}$ or appearance of new lesion was determined. If the smallest sum of the diameters was not increased enough to classify as PD or decreased enough to classify as PR, it was accepted as stable disease (SD).

A single radiologist (A.O.) performed the blinded quantitative analysis with the dedicated software (AW Volume Share, GE, USA). An ADC map was created automatically from conventional DWI using all $16 \mathrm{~b}$-values. For the evaluation of IVIM values, the SI was measured from all 16 different b-values. Lesions were identified for each sequence. ROIs were positioned in a correlated location between DWI and IVIM scans, covering at least $2 / 3$ of the volume of a single lesion, avoiding the vascular and biliary structures. The same ROI was propagated to all $16 \mathrm{~b}$-values. The mean size of ROIs was $9.39 \mathrm{~cm}^{2}$ (range: $0.75-54.86 \mathrm{~cm}^{2}$ ) for lesions and $4.16 \mathrm{~cm}^{2}$ (range $3.71-4.81 \mathrm{~cm}^{2}$ ) for normal parenchyma.

For quantitative measurement of IVIM, the SI were calculated from every $16 \mathrm{~b}$-value between 0 and $1,400 \mathrm{~s} / \mathrm{mm}^{2}$. The following formula was used:

$\mathrm{SI}(\mathrm{b}) / \mathrm{SI}(0)=\mathrm{f} \times \exp \left(-\mathrm{b} \times \mathrm{D}^{*}\right)+(1-\mathrm{f}) \times \exp (-\mathrm{b} \times \mathrm{D})$, where $\mathrm{D}$ is the diffusion coefficient, $\mathrm{D}^{*}$ pseudodiffusion coefficient associated with blood flow, and $\mathrm{f}$ the perfusion fraction.

The $\mathrm{D}, \mathrm{D}^{*}$, and f values were post-processed using a free specific software program from the website http://yamarad.umin. ne.jp/ivim/simplex_en.html [14].

\section{Statistical Evaluation}

The lesions were compared at pre-treatment (PT) and 3, 6, and 9 months of therapy to assess the effect of the IVIM parameters and determine how these parameters change during the antiangiogenic therapy. The goodness of fit of the data to a normal distribu- tion was identified by the Kolmogorov-Smirnov test. The differences in the IVIM parameters and changes in these parameters during the therapy were determined by the independent samples $t$ test. All of the statistical evaluations were performed using SPSS version 18.0 (SPSS Inc., Chicago, IL, USA) statistical software.

\section{Results}

Of the 8 patients with 16 liver metastases included in the study, 4 patients had SD (2 adenocarcinoma lesion, 2 mucinous carcinoma lesion), 3 had PR (11 adenocarcinoma lesion, 2 mucinous carcinoma lesion), and 1 had PD (1 mucinous carcinoma lesion) according to the RECIST 1.1 criteria [13]. Five patients were male, and the mean age was 60 years (range $41-73$ years). Thirteen lesions were adenocarcinoma metastases (11 lesions, 3 lesions were mucinous carcinoma metastases).

Results of the quantitative analysis of IVIM parameters and comparison of PT values with post-treatment values after 3, 6, and 9 months are presented in Table 1 and Figure $2 \mathrm{a}$. The diameters of the metastases were statistically significantly diminished at 9 months when compared with PT $(p=0.03)$. The conventional ADC and D values of the metastases increased at 9 months compared with the PT values ( $p=0.10$, and $p=0.21$, respectively). The $D^{*}$ value was the highest at 3 months $(p=0.24)$, and a decrease was found at 6 and 9 months of therapy $(p=$ 0.97 , and $p=0.87$, respectively). The f value peaked at 3 
Fig. 2. a Time-related measurements of IVIM parameters. b Time-related mean value of signal intensity measurements with increasing $b$-values. PT, pre-treatment; M3, 3rd month; M6, 6th month; M9, 9th month.

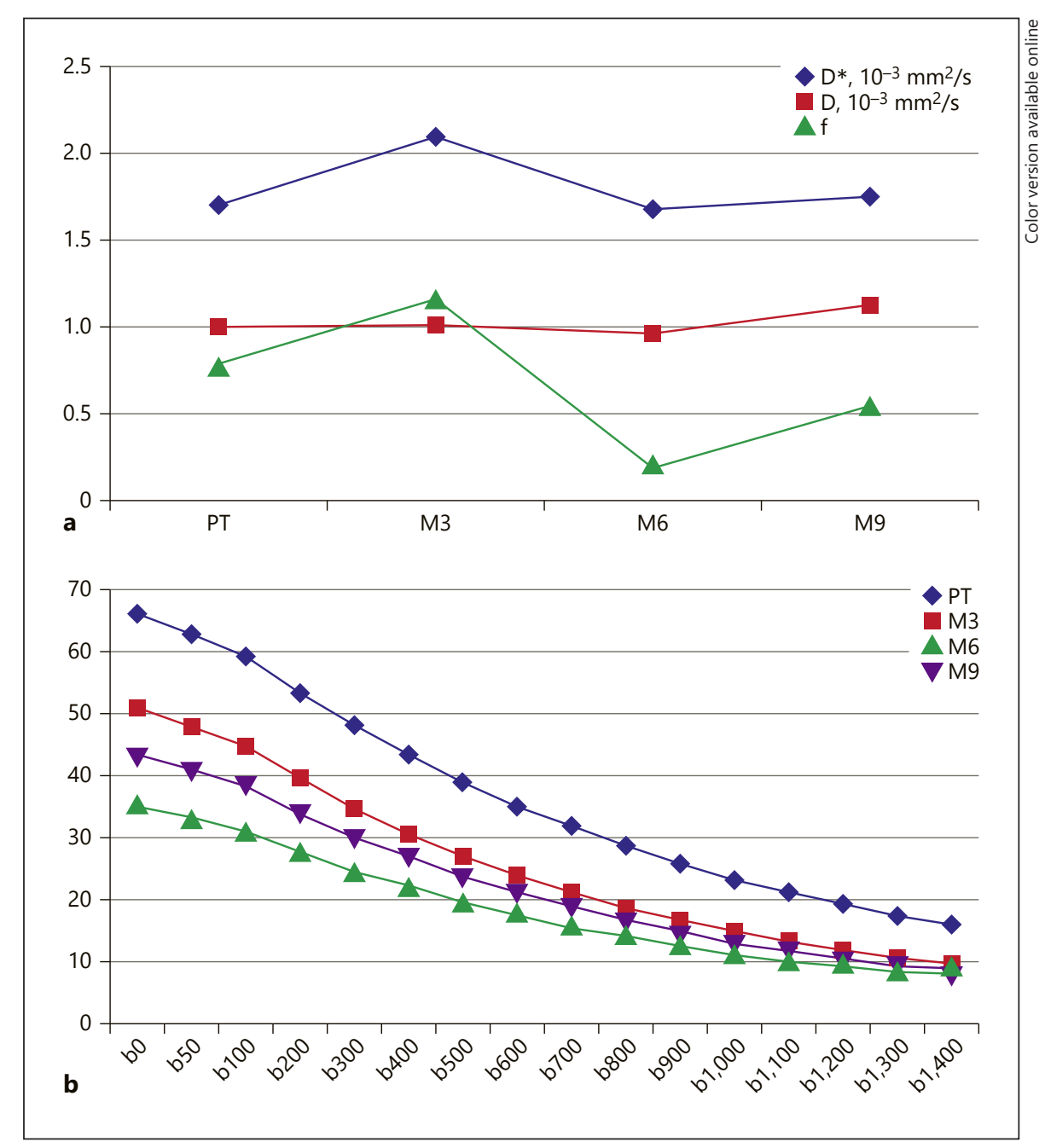

months $(p=0.51)$ and started to decrease thereafter. At 6 months, $\mathrm{f}$ decreased to the lowest values $(p=0.12)$ (Fig. 2b). SI measurements were lowest at 6 months of therapy. IVIM parameter trends for the different disease response groups are summarized in Table 2.

\section{Discussion}

The results of this initial data showed the role of functional IVIM in the assessment of antiangiogenic therapy response without using intravenous contrast agent.

Most studies evaluating the short-term effects of antiangiogenic therapy have shown that successful treatment is reflected by an increase in tumor ADC values [3]. Cui et al. [15] reported that ADC values of responder liver metastases increased after 1 week of chemotherapy. Significant $\mathrm{ADC}$ elevation in responding metastases after long-term therapies has been shown [16]. Granata et al. [3] evaluated the early response to bevacizumab therapy on the 14th day of therapy, with decreased $\mathrm{f}$ values prior to $\mathrm{ADC}$ changes. In our study, $\mathrm{ADC}$ and $\mathrm{D}$ values of the liver metastases increased 9 months after the onset of therapy, reflecting the long-term therapy-induced necrosis within the metastases.

Lower perfusion fraction ( $\mathrm{f}$ ) and $\mathrm{D}^{*}$ values compared to normal liver parenchyma have been described for malignant lesions. The $\mathrm{f}$ and $\mathrm{D}^{*}$ values have been shown to be significantly correlated with the microvessel density score and microenvironment of the tumor and provide information on tumor perfusion, and that lower $f$ values in tumors correlate with lower histological microvessel density [17]. It has been reported that low $\mathrm{D}^{*}$ values are associated with immature and leaky blood vessels, causing high lesional interstitial fluid pressure, resulting in stagnant blood flow [18]. In our study, the f and $\mathrm{D}^{*}$ values 
Table 2. Quantitative analysis of IVIM parameters for the different disease response groups

\begin{tabular}{llllll}
\hline & & $\mathrm{ADC}, 10^{-3} \mathrm{~mm}^{2} / \mathrm{s}$ & $\mathrm{D}, 10^{-3} \mathrm{~mm}^{2} / \mathrm{s}$ & $\mathrm{D}, 10^{-3} \mathrm{~mm}^{2} / \mathrm{s}$ & $\mathrm{f}$ \\
\hline PT & PR & $0.87 \pm 0.40$ & $1.42 \pm 2.96$ & $0.86 \pm 0.43$ & $0.60 \pm 3.06$ \\
& SD & $1.35 \pm 0.32$ & $1.53 \pm 0.51$ & $1.42 \pm 0.48$ & $1.14 \pm 2.23$ \\
& PD & 0.84 & 0.59 & 0.84 & 0.0063 \\
\hline 3 months & PR & $0.92 \pm 0.90$ & $2.50 \pm 7.77$ & $0.83 \pm 0.99$ & $1.42 \pm 2.96$ \\
& SD & $1.50 \pm 0.12$ & $2.02 \pm 0.64$ & $1.50 \pm 0.12$ & $1.27 \pm 1.26$ \\
& PD & 1 & 0.97 & 0.99 & 0.66 \\
\hline 6 months & PR & $0.87 \pm 54$ & $1.91 \pm 2.47$ & $0.87 \pm 54$ & $0.34 \pm 2.21$ \\
& SD & $1.35 \pm 0.14$ & $1.71 \pm 0.28$ & $1.34 \pm 0.14$ & 0.20 \\
& PD & - & - & - & - \\
\hline 9 months & PR & $1.13 \pm 0.26$ & $1.98 \pm 5.01$ & $1.12 \pm 0.27$ & $0.75 \pm 1.41$ \\
& SD & $1.19 \pm 0.05$ & $0.75 \pm 0.11$ & $1.17 \pm 0.06$ & $0.02 \pm 0.17$ \\
& PD & - & - & - & - \\
\hline
\end{tabular}

Mean pre-treatment (PT) values and values at 3, 6, and 9 months post-treatment in the liver metastases. ADC, apparent diffusion coefficient; IVIM, intravoxel incoherent motion; $\mathrm{f}$, perfusion fraction; $\mathrm{D}^{*}$, pseudodiffusion coefficient; D, true diffusion coefficient ; PR, partial response; SD, stable disease; PD, progressive disease.

started to decrease after 3 months of therapy. This might suggest that in the first 3 months, normal vascularization caused by antiangiogenic effects of therapy was accomplished, and that after 3 months the destruction of the normal vascularization had started.

Both cellular apoptosis and vascular perfusion changes after chemotherapy affect the lesion size [19]. In our study, the diameters of the metastases were significantly smaller after 9 months from the start of the therapy when compared to PT. According to the RECIST 1.1 criteria, 3 patients had PR, 4 patients had SD, and 1 patient had PD. The patient with PD and 2 patients with SD had mucinous-subtype carcinoma. The relationship between mucinous carcinomas and evaluation of antiangiogenic therapy warrants further investigation, as our study did not allow this due to the small number of included patients.

Also, the number and the values of $b$, affect the quality of the IVIM parameters. Recent abdominopelvic IVIM studies have used $b$-values between 3 and 35 as there is no standardized protocol [20]. The use of an increased number of b-values leads to extended acquisition time and motion artefacts. Respiratory triggered sequences can reduce the respiratory motion artefacts [21]. Although we used respiratory navigation, we excluded 3 patients due to motion-related artefacts. Dyvorne et al. [22] used only four b-values $(0,15,150$, and 800$)$ and reduced the scan time by up to $75 \%$ and reduced motion related artefacts.

The limitations of our study include the small number of patients, imaging sequence-related distortions, and limitations in the configuration of the IVIM mathematical model. The intra- and interobserver variability was not assessed. In a recent study by Sun et al. [23] in rectal cancer patients, no significant intra- or interobserver difference was detected in the measurement of IVIM parameters of the same DWI scan.

\section{Conclusion}

IVIM is a diffusion imaging method which is being used clinically for evaluation of tissue perfusion without the use of contrast media. We suggest that IVIM could be added in routine MRI protocols in unresectable mCRC patients with liver metastasis to assess the tumor response to antiangiogenic agents. The f value of IVIM may reflect the response of antiangiogenic therapy quantitatively at the earliest interval. The effects of antiangiogenic therapy became measurable with IVIM after 3 months of therapy. IVIM performance in other types of cancer with different organ metastases merits further investigation in larger populations.

\section{Statement of Ethics}

This research complies with the guidelines for human studies and was conducted ethically in accordance with the World Medical Association Declaration of Helsinki. 


\section{Disclosure Statement}

All authors declare that they have no conflicts of interest.

\section{Funding Sources}

The study had no sponsors or funding source.

\section{Author Contributions}

Conception and design of the work, acquisition, analysis, and interpretation of data: Öz A, Server S, Sökmen BK, Namal E, İnan N, Balc1 NC. Drafting the manuscript and critical revision for intellectual content: Öz A, İnan N, Balcı NC. Final perusal and approval of the manuscript: Öz A, İnan N, Balcı NC.

\section{References}

1 Thirion P, Michiels S, Pignon JP, Buyse M, Braud AC, Carlson RW, et al.; Meta-Analysis Group in Cancer. Modulation of fluorouracil by leucovorin in patients with advanced colorectal cancer: an updated meta-analysis. I Clin Oncol. 2004 Sep;22(18):3766-75.

2 Fuchs CS, Marshall J, Barrueco J. Randomized, controlled trial of irinotecan plus infusional, bolus, or oral fluoropyrimidines in first-line treatment of metastatic colorectal cancer: updated results from the BICC-C study. J Clin Oncol. 2008 Feb;26(4):689-90.

3 Granata V, Fusco R, Catalano O, Filice S, Amato DM, Nasti G, et al. Early assessment of colorectal cancer patients with liver metastases treated with antiangiogenic drugs: the role of Intravoxel Incoherent Motion in Diffusion-Weighted Imaging. PLoS One. 2015 Nov;10(11):e0142876.

4 Van Cutsem E, Cervantes A, Nordlinger B, Arnold D; ESMO Guidelines Working Group. Metastatic colorectal cancer: ESMO Clinical Practice Guidelines for diagnosis, treatment and follow-up. Ann Oncol. 2014 Sep;25 Suppl 3:iii1-9.

5 Anzidei M, Napoli A, Zaccagna F, Cartocci G, Saba L, Menichini G, et al. Liver metastases from colorectal cancer treated with conventional and antiangiogenetic chemotherapy: evaluation with liver computed tomography perfusion and magnetic resonance diffusionweighted imaging. J Comput Assist Tomogr. 2011 Nov-Dec;35(6):690-6.

6 Hong SI, Ahn S, Lee YS, Kim WY, Lim KS, Lee $\mathrm{JH}$, et al. Contrast-induced nephropathy in patients with active cancer undergoing contrast-enhanced computed tomography. Support Care Cancer. 2016 Mar;24(3):1011-7.

7 Penner AH, Sprinkart AM, Kukuk GM, Gütgemann I, Gieseke J, Schild $\mathrm{HH}$, et al. Intravoxel incoherent motion model-based liver lesion characterisation from three b-value diffusion-weighted MRI. Eur Radiol. 2013 Oct; 23(10):2773-83.
8 Koh DM, Collins DJ, Orton MR. Intravoxel incoherent motion in body diffusion-weighted MRI: reality and challenges. AJR Am J Roentgenol. 2011 Jun;196(6):1351-61.

9 Le Bihan D, Breton E, Lallemand D, Aubin ML, Vignaud J, Laval-Jeantet M. Separation of diffusion and perfusion in intravoxel incoherent motion MR imaging. Radiology. 1988 Aug;168(2):497-505.

10 Yang SH, Lin J, Lu F, Han ZH, Fu CX, Lv P, et al. Evaluation of antiangiogenic and antiproliferative effects of sorafenib by sequential histology and intravoxel incoherent motion diffusion-weighted imaging in an orthotopic hepatocellular carcinoma xenograft model. J Magn Reson Imaging. 2017 Jan;45(1):270-80.

11 Shi C, Liu D, Xiao Z, Zhang D, Liu G, Liu G, et al. Monitoring Tumor Response to Antivascular Therapy Using Non-Contrast Intravoxel Incoherent Motion Diffusion-Weighted MRI. Cancer Res. 2017 Jul;77(13):3491501.

12 Yang J, Liao C, Liu Y, Yang G, Ke T, Ding Y, et al. MR imaging biomarkers evaluating vascular normalization window after anti-vessel treatment. Oncotarget. 2017 Nov;9(15): 11964-76.

13 Eisenhauer EA, Therasse P, Bogaerts J, Schwartz LH, Sargent D, Ford R, et al. New response evaluation criteria in solid tumours: revised RECIST guideline (version 1.1). Eur J Cancer. 2009 Jan;45(2):228-47.

14 Department of Radiology. University of Yamanashi. Measurement of diffusion parameters with IVIM method. Available from: http://yamarad.umin.ne.jp/ivim/simplex_ en.html

15 Cui Y, Zhang XP, Sun YS, Tang L, Shen L. Apparent diffusion coefficient: potential imaging biomarker for prediction and early detection of response to chemotherapy in hepatic metastases. Radiology. 2008 Sep;248(3):894900 .
16 Koh DM, Scurr E, Collins D, Kanber B, Norman A, Leach MO, et al. Predicting response of colorectal hepatic metastasis: value of pretreatment apparent diffusion coefficients. AJR Am J Roentgenol. 2007 Apr;188(4): 1001-8.

17 Lee HJ, Rha SY, Chung YE, Shim HS, Kim YJ Hur J, et al. Tumor perfusion-related parameter of diffusion-weighted magnetic resonance imaging: correlation with histological microvessel density. Magn Reson Med. 2014 Apr;71(4):1554-8.

18 Kim S, Decarlo L, Cho GY, Jensen JH, Sodickson DK, Moy L, et al. Interstitial fluid pressure correlates with intravoxel incoherent motion imaging metrics in a mouse mammary carcinoma model. NMR Biomed. 2012 May;25(5): 787-94.

19 Li SP, Padhani AR. Tumor response assessments with diffusion and perfusion MRI. J Magn Reson Imaging. 2012 Apr;35(4):74563.

20 Lemke A, Stieltjes B, Schad LR, Laun FB. Toward an optimal distribution of $\mathrm{b}$ values for intravoxel incoherent motion imaging. Magn Reson Imaging. 2011 Jul;29(6):766-76.

21 Aumann EK, Server S, Koyuncu Sokmen B, Oz A, Namal E, Gurcan NI, et al. Diagnostic performances of intravoxel incoherent motion and conventional diffusion-weighted imaging in the differential diagnosis of benign and malignant portal vein thrombus. Abdom Radiol (NY). 2018 Sep;43(9):2270-6.

22 Dyvorne H, Jajamovich G, Kakite S, Kuehn B, Taouli B. Intravoxel incoherent motion diffusion imaging of the liver: optimal b-value subsampling and impact on parameter precision and reproducibility. Eur J Radiol. 2014 Dec; 83(12):2109-13

23 Sun $\mathrm{H}, \mathrm{Xu} \mathrm{Y,} \mathrm{Xu} \mathrm{Q,} \mathrm{Shi} \mathrm{K,} \mathrm{Wang} \mathrm{W.} \mathrm{Rectal}$ cancer: Short-term reproducibility of intravoxel incoherent motion parameters in $3.0 \mathrm{~T}$ magnetic resonance imaging. Medicine (Baltimore) 2017;96:e 68 